\title{
CONTROLE RESTRITO EM UMA TAREFA DE MATCHING-TO-SAMPLE COM PALAVRAS E SILLABAS: AVALIAÇÃO DO DESEMPENHO DE UMA CRIANÇA DIAGNOSTICADA COM AUTISMO ${ }^{1}$
}

\author{
RESTRICTED CONTROL IN A TASK OF MATCHING TO SAMPLE WITH WORDS AND \\ SYLLABLE: EVALUATION OF THE PERFORMANCE OF A CHILD WITH AUTISM
}

\author{
Cássia leal da Hora e Marcelo Frota lobato Benvenuti \\ PONTIFÍCIA UNIVERSIDADE CATÓLICA DE SÃO PAULO, BRASIL
}

\begin{abstract}
RESUMO
Os objetivos do presente estudo foram (1) identificar controle restrito de estímulos no desempenho de um menino autista em uma tarefa de matching-to-sample (MTS) que utilizou sílabas e palavras como estímulos e (2) avaliar um procedimento conhecido como resposta de observação diferencial (DOR), que impedia que o responder da criança aos estímulos comparação fosse baseado em apenas uma das sílabas da palavra apresentada como modelo. Foram conduzidas sessōes de pré-teste em tarefas de MTS simultâneo (SMTS) ou com atraso (DMTS). Precisão em nível intermediários em tarefas de DMTS palavra/sílaba ou em tarefas SMTS palavra/palavra indicou que o participante respondia aos estímulos comparação com base em apenas uma das sílabas da palavra apresentada como modelo. A análise dos erros mostrou que, em geral, o participante respondia sob controle da primeira sílaba, escolhendo qualquer uma das palavras apresentadas como estímulo-comparação que tivesse a mesma primeira sílaba da palavra apresentada como modelo. Posteriormente, a precisão em linha de base DMTS palavras/sílabas foi comparada com a precisão em uma tarefa em que tentativas SMTS palavra/palavra eram intercaladas com tentativas DMTS palavras/sílabas. Os resultados indicaram que durante a vigência do procedimento com tentativas DMTS intercaladas às tentativas SMTS não ocorreu correção completa do controle restrito. No entanto, com o retorno à linha de base, observou-se a melhora no desempenho do participante, indicando a diminuição do controle restrito e mudança no padrão de erro.
\end{abstract}

Palavras-chave: controle restrito de estímulos; resposta de observação; matching-to-sample; autismo.

\begin{abstract}
The aims of the present study were (1) identifying restricted stimuli control at performance of an autistic sixyear-old-boy in a matching-to-sample task (MTS) that used syllables and words as stimuli and (2) assessing a procedure, known as differential observing response (DOR), which not allowed the child to respond to a comparison stimuli based on just one syllable of the word presented as model. Pre-tests sessions were conducted with simultaneous or delay MTS tasks with syllables and words. The accuracy on intermediate levels in DMTS word/syllables or in SMTS word/word indicated that the participant chose based on one of the syllables of the sample stimulus, but not based on both of them. The analysis of the errors showed that, usually, the participant responded under control of the first syllable, choosing a word, presented as a comparison, which contained the first syllable of the model. Accuracy at the baseline was compared with the precision in a task that required that participant's responses were based, in separated trials, on both syllables of the compound stimulus. The results indicated that, during the DMTS procedure with SMTS word/word trials, the restricted control wasn't completely reduced. However, with the return to the baseline, there was improvement in the participant's performance, indicating a decrease of restricted control and changes in the pattern of error.
\end{abstract}

Key-words: restricted stimulus control, observing response; matching-to-sample; autism.

\footnotetext{
1 Artigo resultado da pesquisa intitulada "Avaliação de um procedimento de resposta de observação para identificar e corrigir controle restrito de estímulos em uma tarefa de leitura com uma criança diagnosticada com autismo", realizada no período de 2005 a 2006 com financiamento FAPESP na forma de bolsa de Iniciação Científica para a primeira autora. Os autores agradecem as contribuições do Professor Gerson Tomanari por disponibilizar o computador para a coleta de dados e novamente ao Professor Gerson Tomanari e à Professora Paula Debert pela possibilidade de discussão de uma primeira versão deste trabalho no grupo de pesquisa do Laboratório de Análise Experimental do Comportamento do Instituto de Psicologia da Universidade de São Paulo. Endereço para correspondência: Cássia Leal da Hora, Al. Ribeirão Preto, 163 apt.38. - Bela Vista, São Paulo - SP, Cep: 01133-001. Endereço eletrônico: cassia_hora@uol.com.br.
} 
Controle restrito de estímulos pode ser identificado quando, após uma história de reforço diferencial, ocorre o controle por um número limitado exercido pelos elementos de um estímulo antecedente (Litrownik, McInnis, Wetzel-Pritchard, \& Filipelli, 1978). Lovaas, Schreibman, Koegel e Rehm (1971) usaram a expressão hiperseletividade de estímulos em um estudo no qual definiram esse problema como o modo pelo qual crianças respondem sob controle atipicamente limitado com respeito à extensão, amplitude ou número de características de um estímulo.

O estabelecimento de controle restrito é um problema freqüente no ensino de indivíduos com desenvolvimento atípico, em especial os que recebem o diagnóstico de autismo. Freqüentemente, essas crianças ficam sob controle de aspectos irrelevantes dos estímulos que são apresentados em seu ambiente (Allen \& Fuqua, 1985; Dube, Lombard, Farren, Flusser, Balsamo, Fowler \& Tomanari, 2003; Koegel \& Koegel, 1995). Controle restrito pode ser considerado um exemplo de falta de coerência de topografia de controle de estímulos: certos controles são estabelecidos pelo reforço, mas não exatamente aqueles planejados pelo experimentador (Serna, Mcllvane, Lionello-DeNolf, Barros \& Dube, 2000). Na aprendizagem de leitura, a falta de coerência de controle de estímulos pode ser um problema especial (p. ex., Birnie-Selwyn \& Guerin, 1997). Ao aprender a palavra BOLO a criança pode responder apenas sob controle da sílaba BO. Na medida em que o controle restrito permaneça, é impossível a leitura correta posterior de outras palavras que contenham a mesma sílaba, por exemplo, a palavra BOCA. Diante desta, a criança continuará a ler BOLO.

Controle restrito de estímulos pode ser facilitado por certas estratégias de ensino, geralmente eficazes para indivíduos com ou sem déficits comportamentais (Lovaas, Koegel, \& Schreibman, 1979). Contudo, a análise do comportamento tem feito muitos avanços na elaboração de procedimentos para correção de controle de estímulos que prejudica aprendizagens mais complexas, com indivíduos com ou sem atrasos no desenvolvimento.

Entre os esforços para reduzir o controle restrito de estímulos, Dube e McIlvane (1999) investigaram os efeitos de um procedimento denominado resposta de observação diferencial (DOR) para corrigir o estabelecimento de controle restrito em indivíduos com retardo mental moderado. Os participantes do estudo foram submetidos a diferentes tipos de pré-testes de matching-to-sample (MTS) com estímulos compostos abstratos ou componentes do estímulo composto (estímulos simples). Nos pré-testes em que estímulos-modelo e comparações eram simples, os participantes apresentaram precisão próxima ou acima de 90\%. Nas tarefas em que o estímulo-modelo era composto e os estímulos-comparação eram os componentes do estímulo composto (ora um, ora outro), um desempenho com alta precisão indicava a ausência de controle restrito. No outro extremo, precisão próxima de níveis do acaso (33\%, considerando a apresentação de três estímulos-comparação) indicava uma falha global para o desempenho na tarefa de matching. Resultados de precisão intermediária (p.ex., 67\%) indicavam controle restrito, isto é, controle exercido por apenas um dos componentes do estímulo composto: diante de determinado estímulo modelo composto, o participante acertava todas as vezes em que era apresentado um dos seus componentes como comparação correto. Quando era apresentado o outro componente como comparação correto, 
acertos aconteciam ao nível do acaso. Em uma tarefa de MTS com três comparações, o resultado envolvendo esse controle produz uma precisão intermediária acima da porcentagem de $50 \%$ de acerto e abaixo da de $90 \%$.

Os resultados do pré-teste do estudo de Dube e McIlvane (1999) mostraram que os participantes apresentaram desempenhos em nível intermediário, indicativo de controle restrito, nas tarefas de MTS com atraso quando os estímulosmodelo/comparação eram composto/simples (estímulo-modelo composto e comparações simples). Em seguida, os níveis de precisão da condição de linha de base foram comparados com os resultados após a introdução de um procedimento que exigia dos participantes uma resposta de observação diferencial (DOR). Na condição DOR, tentativas de DMTS compostos/ simples foram intercaladas com tentativas MTS composto/composto. Quando respostas de observação foram requeridas, o nível de precisão aumentou. No retorno à condição de linha de base, com a tarefa MTS compostos/simples, a precisão retornou aos níveis intermediários.

Walpole, Roscoe e Dube (2007) utilizaram recentemente o procedimento de resposta de observação diferencial em uma situação de ensino de pré-requisitos de leitura com uma criança autista. O participante apresentava desempenho acurado em tarefas de MTS de identidade em que eram utilizados como estímulos palavras impressas de três letras, sem letras em comum. Quando, em outra condição, as palavras envolviam letras em comum, o desempenho era o típico de responder ao nível do acaso. $\mathrm{O}$ procedimento de correção, com palavras com letras em comum, envolveu a apresentação das palavras como estímulos-modelo; como estímuloscomparação, eram apresentadas letras críticas que diferenciavam as palavras. $\mathrm{O}$ reforço era contingente à escolha da letra que estivesse presente na palavra apresentada como modelo. Por exemplo, com as palavras "cat", "can” e "car", as tentativas consistiam na apresentação das palavras como modelo e a sílaba " $\mathrm{t}$ ", " $\mathrm{n}$ " ou " $r$ " como comparações. Diante do modelo "cat" a criança era reforçada se escolhesse " $t$ ". Com esse procedimento, a quantidade de acerto aumentou e permaneceu alta, com índices de cerca de $100 \%$ de acerto, mesmo com a remoção do procedimento de correção.

Palavras são estímulos compostos que podem gerar controle restrito em relação a diferentes aspectos. A letra crítica de palavras com letras em comum pode ser um desses aspectos. Controle restrito também pode envolver as sílabas das palavras. Identificar e corrigir controle restrito envolvendo sílabas de palavras pode ser especialmente importante se considerarmos que a recombinação de sílabas é uma das maneiras pelas quais a capacidade de ler palavras novas, não explicitamente treinadas, é avaliada em crianças que passam por programas de leitura baseados nos procedimentos da análise do comportamento (ver, por exemplo, de Rose, de Souza, Rossito, \& de Rose, 1989; Hanna, de Souza, de Rose, \& Fonseca, 2004; Matos, Hübner \& Peres, 1997).

O presente estudo avalia o problema do controle restrito de estímulos em uma criança diagnosticada com autismo. O objetivo foi verificar se é possível identificar controle restrito de estímulos no desempenho da criança em uma tarefa de MTS em que foram utilizadas sílabas como estímulos simples e palavras formadas pelas mesmas sílabas como estímulos compostos. Para identificação e correção do controle restrito nessas condiçôes foi utilizado o procedimento de correção proposto por Dube e McIlvane (1999). 
MÉTodo

\section{Participantes}

Um menino de 6 anos, diagnosticado com autismo (F.). O participante era submetido à Intervenção Comportamental há aproximadamente três anos por uma equipe de psicólogos de uma clínica particular. A intervenção visava desenvolver repertório comportamental, como comunicação e habilidades pré-acadêmicas, bem como minimizar comportamentos incompatíveis com a aprendizagem ou que produzissem danos diretos ou indiretos para a criança. Como parte dos procedimentos para aquisição de habilidades acadêmicas durante o desenvolvimento do estudo, F. também foi submetido a um programa de pré-alfabetização (de Rose et al. 1989) que utilizava parte das palavras selecionadas como estímulos neste estudo. O diagnóstico de autismo foi realizado por um psiquiatra infantil com bases nos critérios diagnósticos do DSM-IV. Os responsáveis de F. demonstraram interesse em colaborar com a pesquisa por meio da assinatura de termo de Consentimento Livre e Esclarecido, aprovado pelo comitê de ética em pesquisa da Universidade em que foi realizada a pesquisa (em conformidade com os critérios da Resolução no 196 de 10 de outubro de 1996 do Conselho Nacional de Saúde do Ministério da Saúde).

\section{Local equipamento}

As sessões experimentais de 10 a 25 minutos foram conduzidas em uma sala bastante freqüentada pela criança na sua casa. Quando a criança está em casa, ela passa a maior parte do tempo na sala, pois é o ambiente em que assiste televisão, brinca e faz as refeiçôes principais do dia-a-dia. Durante a coleta de dados, apenas a pesquisadora e a criança permaneciam no local. Um computador Apple Machintosh com tela sensível ao toque (MicroTouch's ADB touchscreen) ficava em cima da mesa de jantar e controlava a apresentação de estímulos, registro de respostas e dados coletados através do software MTS v. 11.4.6 (Dube \& Hiris, 1999).

A tela do computador possuía cinco chaves de apresentação de estímulos. Cada uma delas media 136 pixels. A chave de apresentação do modelo ficava localizada no centro da tela e as chaves dos estímulos-comparação ficavam próximas aos quatro cantos do monitor. $\mathrm{O}$ participante ficou sentado em uma cadeira, em frente ao computador, de onde podia tocar a tela do monitor. A pesquisadora ficou sentada em uma cadeira ao lado do participante, de forma que podia interagir com a criança e manipular o equipamento quando necessário.

Foram utilizados como estímulos compostos palavras com duas sílabas (BOLO, TATU, VELA); sílabas isoladas foram consideradas estímulos simples (BO, LO, VE, LA, TA, TU). Os estímulos tinham a cor preta, fonte Geneva, tamanho $36 \mathrm{e}$ foram apresentados ao participante em uma tela de fundo branco. A coleta de dados foi realizada no mínimo uma vez por semana e no máximo três, em dias alternados. A quantidade de dias em que ocorriam as coletas semanais foi definida a partir da disponibilidade do participante. Em cada dia, eram realizadas uma ou mais sessōes, a depender da etapa do estudo. A quantidade de tentativas de cada sessão será definida a seguir, na descrição de cada etapa.

\section{Procedimento}

Testes de Preferência de Estímulos. Antes do início de cada dia de coleta de dados, a criança era submetida a um teste de preferências de 
estímulos (De Leon \& Iwata, 1996), para avaliação de itens que seriam utilizados como conseqüências para as respostas corretas.

Matching-to-sample (MTS). A tarefa utilizada em todas as fases da pesquisa foi o MTS, atrasado ou simultâneo. Inicialmente, o participante foi submetido a uma avaliação de habilidades em MTS baseada em Dube, Iennaco e McIlvane (1993). A tarefa consistia na apresentação de um estímulo-modelo no centro da tela que devia ser tocada pelo participante para ocorrer a apresentação dos estímulos-comparação. Depois que o participante tocava o modelo, três estímuloscomparação apareciam nos cantos da tela. Nas tarefas de matching simultâneo (SMTS), o estímulo-modelo ficava disponível na tela até que a criança tocasse um dos estímuloscomparação. Nas tarefas matching atrasado (DMTS), o estímulo-modelo desaparecia da tela assim que o participante o tocava e os estímulos-comparação apareciam imediatamente depois do desaparecimento do estímulo-modelo ( 0 s de atraso). Respostas em espaços fora das chaves de apresentação de estímulos não recebiam nenhuma consequiência, mas eram registradas como erro pelo software. Durante os intervalos entre as tentativas - IETs de 3 segundos -, a tela toda ficava em branco.

Respostas corretas eram seguidas por uma breve apresentação auditivo-visual na tela do computador, além de por uma conseqüência tangível escolhida pelo participante no teste de preferências. Respostas incorretas eram seguidas apenas de IET. Em algumas fases do estudo, a conseqüência tangível era apresentada a cada resposta correta; em outras, o participante tinha acesso ao item tangível após o acúmulo de cinco fichas (em uma condição denominada "acúmulo de fichas"). Respostas ao estímulo-modelo depois de apresentados os estímulos-comparação não tiveram qualquer conseqüência programada.

Fase 1 - Pré-testes de SMTS e DMTS de identidade. Durante esta fase, respostas corretas consistiam na escolha de um estímulocomparação que mantivesse identidade com o estímulo apresentado como modelo ou na escolha de um estímulo comparação que era uma sílaba da palavra apresentada como modelo. As sessões eram realizadas até que o desempenho do participante ficasse estável. O critério para estabilização foi precisão acima de $50 \%$ em três sessôes consecutivas, desde que a precisão de cada sessão individual não desviasse da média das três sessões em um valor maior de 10\%. O número de tentativas das sessões, para cada tipo de pré-teste, corresponde às combinaçôes possíveis entre um estímulomodelo (simples ou composto) e todas as combinações entre um estímulo-comparação correto e dois incorretos. Essas combinações podem ser conferidas nas tabelas apresentadas na seção de Resultados.

Os seguintes tipos de pré-testes foram realizados:

1) SMTS sílaba-silaba: Cada sessão foi composta de 30 tentativas de matching de identidade simultâneo, nas quais estímulosmodelo e comparações eram sílabas. Nesta e na próxima condição, os estímulos-comparação incorretos que foram apresentados numa mesma tentativa nunca foram aqueles que nas fases posteriores compuseram uma palavra com o estímulo-modelo (p.ex., na tentativa em que o modelo fosse "BO", nunca havia o estímulocomparação "LO").

2) DMTS sílaba-sílaba: Cada sessão foi composta de 30 tentativas de matching de 
identidade com atraso ( $0 \mathrm{~s}$ de atraso), nas quais estímulos-modelo e comparações eram sílabas.

3) SMTS palavra/silaba. Cada sessão foi composta de 30 tentativas, nas quais o estímulomodelo foi sempre uma palavra. O estímulocomparação correto era uma das duas sílabas apresentadas no estímulo-modelo. Qualquer uma das sílabas da palavra apresentada como modelo tinha uma probabilidade equivalente de aparecer como o estímulo-comparação correto. Em cada tentativa, eram apresentados dois estímuloscomparaçóes incorretos, sílabas diferentes daquelas apresentadas que compunham a palavra apresentada como modelo.

4) DMTS palavralsilaba. Cada sessão foi composta de 30 tentativas, com os mesmos critérios da etapa anterior para preparação das tentativas.

5) SMTS palavralpalavra. Cada sessão foi composta de 45 tentativas. Estímuloscomparação eram duas palavras: uma com a primeira sílaba da palavra apresentada como modelo e outra com a segunda sílaba da palavra apresentada como modelo. Devido a um erro de programação, houve três configuraçōes nas quais os estímulos-comparação incorretos tinham apenas a segunda sílaba idêntica à segunda sílaba do estímulo-modelo. As implicações desse erro serão abordadas na seção de Resultados.

6) SMTS palavra/palavra com acúmulo de fichas. As sessões foram compostas pelo mesmo tipo de tentativa da condição anterior. Os critérios para apresentação de estímuloscomparação incorretos foram os mesmos da etapa anterior. A diferença do procedimento consistiu na mudança da forma como as consequiências reforçadoras eram apresentadas ao participante. A cada resposta correta, F. recebia a conseqüência que já estava programada no computador (som e estrelas) e uma ficha. Após o acúmulo de cinco fichas, a criança recebia algum item escolhido no teste de preferências. O esquema de fichas foi introduzido para evitar a saciação dos itens "preferidos", que na maioria das vezes eram comestíveis (chocolate ou batata frita), freqüentemente escolhidos em primeiro ou segundo lugar no teste de preferências. Caso a criança emitisse uma resposta incorreta, a conseqüência programada era apenas o IET.

\section{Fase 2 - Procedimento de Correção DOR} (Resposta de Observação Diferencial). Esta fase foi planejada para avaliar os efeitos do procedimento de DOR (Resposta de Observação Diferencial) na tarefa de DMTS palavra/sílaba. Foram realizadas sessões de linha de base, DOR e Pós-DOR, descritas a seguir:

1) Linha de Base. Foram realizadas seis sessões, de 30 tentativas cada, com a tarefa de DMTS palavras/sílabas.

2) $D O R$. Nas sessões desta condição, uma tentativa de DMTS palavra/sílaba foi introduzida depois de uma tentativa de SMTS palavra/palavra. Assim, cada tentativa iniciavase com a apresentação de um estímulo-modelo. O primeiro toque no estímulo-modelo produzia a exibição de três outras palavras: a mesma apresentada como modelo e dois estímulos comparação incorretos, um com a primeira sílaba do modelo e o outro com a segunda sílaba do modelo. Em contraste com o procedimento durante os pré-testes, escolha de qualquer um dos estímulos-comparação não recebiam nenhuma conseqüência programada. Depois de selecionado qualquer estímulo-comparação, iniciava-se a tentativa de DMTS palavras/sílabas na qual o mesmo estímulo-modelo era apresentado novamente. Uma resposta a esse estímulo produzia o aparecimento de três 
sílabas, uma delas sendo a primeira ou a segunda sílaba da palavra apresentada como modelo. Reforço era contingente à escolha da sílaba da palavra do modelo na tentativa de DMTS palavras/sílabas. As respostas incorretas eram seguidas de IET. A Figura 1 mostra um diagrama que ilustra uma tentativa DOR.

Sessões com tentativas DOR incluíram tentativas simples SMTS palavra/palavra com conseqüência diferencial, para evitar o enfraquecimento do responder nesse tipo de tentativa em função da não conseqüenciação. As duas primeiras sessões na condição de DOR composto foram montadas com 66 tentativas (60 de DOR, mais 6 de SMTS palavra/palavra). As oito sessões restantes foram realizadas com 33 tentativas (30 de DOR, mais 3 de SMTS palavra/palavra).

3) PÓS-DOR. Foram realizadas seis sessões de retorno à linha de base. As três primeiras sessões foram com esquema de reforçamento em CRF (o reforço era apresentado a cada resposta correta) e as três últimas envolveram o sistema de acúmulo de fichas, de acordo com o qual o participante recebia uma ficha a cada acerto e, após o acúmulo de cinco fichas, recebia o item escolhido no teste de preferência.

\section{Resultados}

A Tabela 1 mostra a porcentagem de acerto do participante na Fase 1, nas tarefas de pré- teste de MTS. Houve menor número de acertos conforme a tarefa foi incluindo palavras no lugar das sílabas e o atraso entre modelo e os estímulos-comparação. A Tabela 1 também mostra que o desempenho do participante na tarefa SMTS palavra/palavra ficou em níveis intermediários quando o esquema de reforçamento em vigor era o de CRF simples para apresentação do item escolhido nos testes de preferência. Com a introdução da situação de acúmulo de fichas, o participante na tarefa SMTS palavra/ palavra chegou perto de atingir $100 \%$ de respostas corretas nas sessões.

A Figura 2 mostra a porcentagem de acertos obtida na Fase 2, nas sessões de linha de base (DMTS palavra/sílaba), DOR (SMTS palavra/palavra e DMTS palavra/sílaba) e PósDOR (DMTS palavra/sílaba). Podemos observar a ocorrência de aumento imediato dos níveis de precisão na fase de DOR em relação ao que vinha sendo obtido na linha de base. Logo depois do aumento inicial, o número de acertos diminuiu nos dois tipos de tentativas da sessão e houve maior variabilidade sessãoa-sessão. Com exceção da sessão 3 da fase DOR, na qual o participante apresentou $100 \%$ de acerto nas tentativas de DMTS, não ocorreu um aumento sistemático na quantidade de acertos nas tentativas com essa tarefa durante a vigência do DOR, o que indicaria eliminação do controle restrito. Após a retirada do procedimento de correção, os níveis de precisão do desempenho do participante

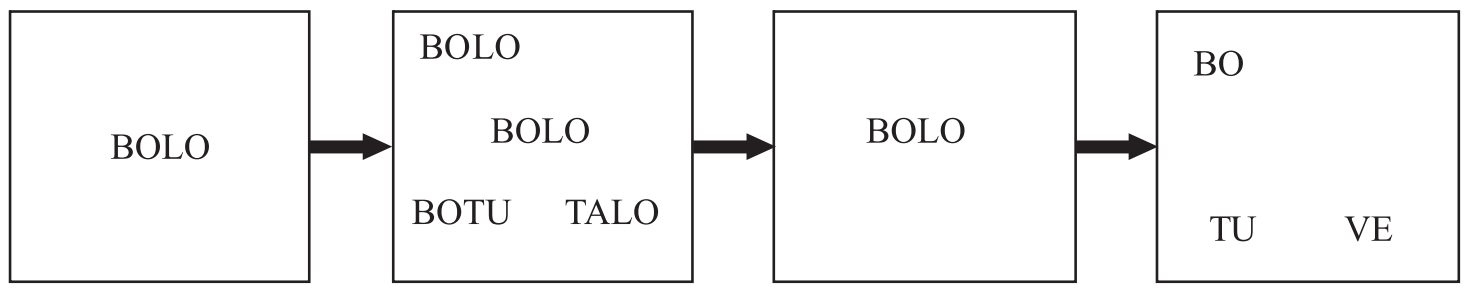

Figura 1. Ilustração da tentativa SMTS palavra/palavra seguida de uma tentativa DMTS palavra/sílaba. A figura mostra as configurações dos estímulos na tela do computador em quatro momentos diferentes. 
Tabela 1

Porcentagem de acerto do participante nas tarefas de MTS do Pré-testes da Fase 1.

\begin{tabular}{cccccccc}
\hline TAREFAS & \multicolumn{7}{c}{ SESSÕES } \\
\cline { 2 - 8 } DE MTS & 1 & 2 & 3 & 4 & 5 & 6 & 7 \\
\hline SMTS sílaba / sílaba & 90 & 97 & 97 & - & - & - & - \\
DMTS sílaba / sílaba & 90 & 87 & 90 & - & - & - & - \\
SMTS palavra / sílaba & 90 & 90 & 83 & - & - & - & - \\
DMTS palavra / sílaba & 70 & 87 & 60 & 76 & 63 & 67 & 63 \\
SMTS palavra / palavra & 60 & 73 & 67 & 53 & 47 & - & - \\
SMTS palavra / palavra & 89 & 98 & 98 & - & - & - & - \\
com acúmulo de fichas & & & & & & & \\
\hline
\end{tabular}

permaneceram no nível intermediário. No entanto, houve mais acertos no Pós-DOR do que durante a linha de base. Assim como observado na Fase 1 para a tarefa de matching simultâneo, a maior quantidade de acertos na tarefa de DMTS da condição Pós-Dor foi observada com a introdução do procedimento de acúmulo de fichas.

Para uma análise mais detalhada do padrão de acertos e erros - sobretudo do padrão de erros, as tabelas a seguir mostram os tipos de tentativas de diferentes condiçôes e a porcentagem de escolhas dos estímuloscomparação, tanto corretos como incorretos. Para facilitar a leitura das tabelas, o número "0\%" diante dos estímulos-comparação incorretos foi omitido quando houve 100\% de acerto diante do $S+$ daquele tipo de tentativa.

A Tabela 2 mostra a porcentagem de respostas do participante a cada estímulo comparação nas tarefas de SMTS e DMTS palavra/sílaba da Fase 1. É possível observar que na tarefa STMS, o participante obteve

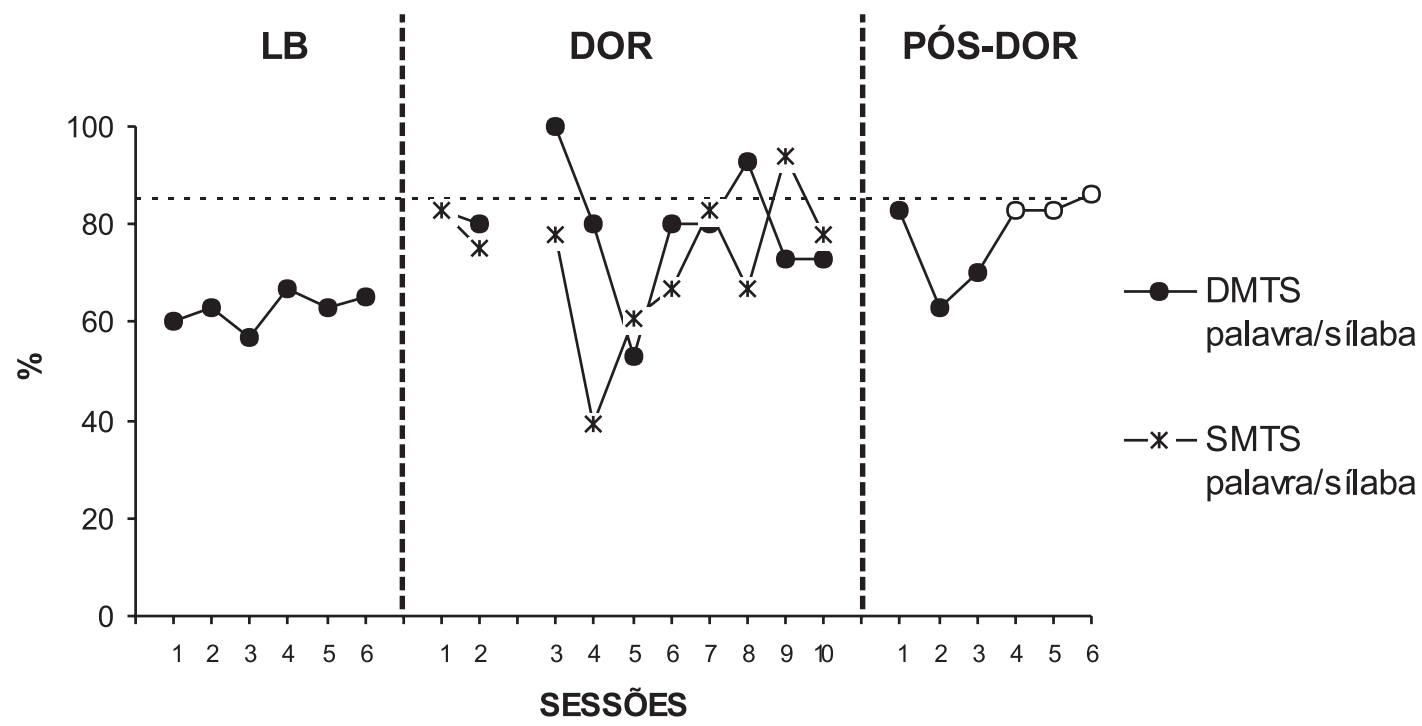

Figura 2. Porcentagem de acertos nas condições de linha de base, DOR e Pós-DOR da Fase 2. 
$100 \%$ de acerto na maioria tentativas. O maior número de erros ocorreu quando o maior número de acertos foi diante do estímulo-modelo era a palavra "TATU” e o estímulo-modelo "BOLO" quando o estímulo-comparação correto era o "TU". estímulo-comparação correto era o "BO". O Nesses casos, houve maior porcentagem de erros

Tabela 2

Porcentagens de escolha dos estímulos-comparação corretos e incorretos nas tarefas SMTS e DMTS palavras/sílabas do Pré-teste de MTS da Fase 1.

\begin{tabular}{|c|c|c|c|c|c|c|c|c|c|}
\hline \multirow[t]{2}{*}{ Estímulo modelo } & \multicolumn{3}{|c|}{ Estímulos de comparação } & \multicolumn{3}{|c|}{ SMTS } & \multicolumn{3}{|c|}{ DMTS } \\
\hline & $S_{+}$ & S- & S- & $S_{+}$ & S- & S- & $\mathrm{S}_{+}$ & S- & S- \\
\hline \multirow{10}{*}{ BOLO } & $\mathrm{BO}$ & TA & $\mathrm{TU}$ & 100 & & & 100 & & \\
\hline & & $\mathrm{TA}$ & VE & 100 & & & 86 & 0 & 14 \\
\hline & & $\mathrm{TA}$ & LA & 100 & & & 100 & & \\
\hline & & VE & LA & 100 & & & 71 & 29 & 0 \\
\hline & & VE & $\mathrm{TU}$ & 100 & & & 86 & 14 & 0 \\
\hline & $\mathrm{LO}$ & $\mathrm{TA}$ & TU & 67 & 0 & 33 & 71 & 14 & 14 \\
\hline & & $\mathrm{TA}$ & $\mathrm{VE}$ & 100 & & & 100 & & \\
\hline & & $\mathrm{TA}$ & LA & 100 & & & 71 & 14 & 14 \\
\hline & & VE & LA & 100 & & & 100 & & \\
\hline & & VE & TU & 100 & & & 86 & 0 & 14 \\
\hline \multirow{10}{*}{ TATU } & $\mathrm{TA}$ & $\mathrm{BO}$ & LO & 100 & & & 57 & 14 & 29 \\
\hline & & $\mathrm{BO}$ & VE & 100 & & & 100 & & \\
\hline & & $\mathrm{BO}$ & LA & 100 & & & 86 & 0 & 14 \\
\hline & & $\mathrm{VE}$ & LA & 67 & 0 & 33 & 86 & 0 & 14 \\
\hline & & $\mathrm{VE}$ & $\mathrm{LO}$ & 100 & & & 71 & 0 & 29 \\
\hline & $\mathrm{TU}$ & $\mathrm{BO}$ & LO & 100 & & & 86 & 0 & 14 \\
\hline & & $\mathrm{BO}$ & VE & 67 & 33 & 0 & 29 & 57 & 29 \\
\hline & & $\mathrm{BO}$ & LA & 33 & 0 & 67 & 14 & 0 & 86 \\
\hline & & VE & LA & 33 & 0 & 67 & 14 & 14 & 71 \\
\hline & & VE & LO & 33 & 33 & 33 & 57 & 14 & 29 \\
\hline \multirow{10}{*}{ VELA } & $\overline{V E}$ & $\mathrm{BO}$ & $\mathrm{LO}$ & 100 & & & 100 & & \\
\hline & & $\mathrm{BO}$ & $\mathrm{TA}$ & 100 & & & 42 & 0 & 57 \\
\hline & & $\mathrm{BO}$ & $\mathrm{TU}$ & 100 & & & 86 & 0 & 14 \\
\hline & & $\mathrm{TA}$ & $\mathrm{TU}$ & 67 & 0 & 33 & 86 & 14 & 0 \\
\hline & & TA & LO & 100 & & & 86 & 14 & 0 \\
\hline & LA & $\mathrm{BO}$ & LO & 100 & & & 71 & 14 & 14 \\
\hline & & $\mathrm{BO}$ & $\mathrm{TA}$ & 100 & & & 29 & 14 & 57 \\
\hline & & $\mathrm{BO}$ & $\mathrm{TU}$ & 100 & & & 86 & 14 & 0 \\
\hline & & $\mathrm{TA}$ & TU & 67 & 0 & 33 & 57 & 14 & 29 \\
\hline & & $\mathrm{TA}$ & LO & 100 & & & 42 & 29 & 29 \\
\hline
\end{tabular}


pela escolha do estímulo-comparação incorreto "LA". Quando o estímulo-comparação correto era o "TA", o participante errou $33 \%$ da vezes pela escolha do "LA". O mesmo padrão de erros diante de "TATU" se repete no pré-teste da tarefa de DMTS. Mais uma vez, a maior porcentagem de erros ocorreu diante do modelo "TATU", especialmente quando o estímulocomparação correto era o "TU”. Outro padrão de erros bastante freqüente na tarefa de DMTS foi a escolha do estímulo-comparação incorreto "TA" nas configuraçōes de estímulos em que o comparação correto era o "LA". Diante de "VELA" foi comum o erro pela escolha de "TA". O maior número de acertos no matching atrasado foi diante do modelo "BOLO" quando o comparação correto era o "BO".

A Tabela 3 mostra a porcentagem de escolhas dos estímulos comparação na linha de base, tentativas DMTS palavra/sílaba da tarefa de DOR e na condição Pós-DOR da Fase 2. O padrão de resposta do participante na tarefa DMTS aproximou-se dos resultados no mesmo tipo de tarefa no pré-teste. Em relação à linha de base e à condição Pós-DOR, houve um número maior de acertos do que de erros e mais erros por escolha das sílabas "TA" e "LA". O participante também apresentou mais acertos quando o modelo era a palavra "BOLO" e o estímulo-comparação correto era a sílaba "BO". Comparando-se a linha de base com a condição de Pós-DOR, é possível notar que na condiçãao final os erros são mais distribuídos entre os diferentes tipos de tentativas e poucas vezes há mais do que $33 \%$ de escolhas de determinado comparação incorreto.

A Tabela 4 mostra a porcentagem de escolhas dos estímulos-comparação corretos e incorretos nas tarefas de DMTS palavra/ palavra comparado com o obtido nas mesmas tentativas do procedimento de DOR. Podemos observar que há uma porcentagem maior de acertos do que de erros tanto na fase de préteste quanto na fase de correção. Durante o pré-teste, o desempenho do participante ficou em nível intermediário em praticamente todas as configurações de estímulos. Houve uma porcentagem maior de erros nos estímulos-comparação incorretos que tinham a primeira sílaba idêntica à primeira sílaba do estímulo-modelo. Por exemplo, do total das respostas incorretas emitidas quando o modelo era a palavra "BOLO", uma grande porcentagem foi emitida em comparações que continham a primeira sílaba do modelo (BOLA e BOTU). Evidência do forte controle exercido pela primeira sílaba do modelo para a escolha do estímulo-comparação, durante o pré-teste, é o resultado nas tentativas em que a configuração estava errada, sem um estímulo-comparação incorreto com a primeira sílaba do modelo: nesses casos, foram obtidos porcentagens de acertos de 73, $80 \mathrm{e}$ $93 \%$ no pré-teste (sinalizadas com ${ }^{*}$ ). No mesmo tipo de tentativa da condição DOR, parece haver uma mudança, ainda que não sistemática, no padrão de erro: há redução no número de erros pela escolha do estímulocomparação com a primeira sílaba do modelo e aumento no número de erros pela escolha do estímulo-comparação incorreto com a segunda sílaba do modelo.

A análise dos resultados da Tabela 4 sugere uma mudança no padrão de erro nas tentativas SMTS palavra/palavra da condição DOR, em relação ao obtido na mesma tarefa durante o pré-teste. Essa mudança de padrão de erro pode ser vista com mais clareza acumulando-se o tipo de erro cometido diante de cada comparação (erro pela escolha do estímulo-comparação com a primeira sílaba e 
erro pela escolha do estímulo-comparação com mostra o número acumulado de escolhas de a segunda sílaba do modelo) ao longo das cada tipo de estímulo-comparação nas sessões sessões da condição SMTS palavra/palavra do de pré-teste SMTS palavra/palavra: escolha do pré-teste e da condição DOR. A Figura 3 estímulo-comparação correto, escolha do Tabela 3

Porcentagem de escolhas dos estímulos-comparação nas tentativas DMTS palavra-sílaba da Fase 2.

\begin{tabular}{|c|c|c|c|c|c|c|c|c|c|c|c|c|}
\hline \multirow[t]{2}{*}{ Estímulo modelo } & \multicolumn{3}{|c|}{$\begin{array}{l}\text { Estímulos } \\
\text { Comparação }\end{array}$} & \multicolumn{3}{|c|}{ Linha de Base } & \multicolumn{3}{|c|}{ DOR } & \multicolumn{3}{|c|}{ Pós-DOR } \\
\hline & $\mathrm{S}_{+}$ & S- & $\overline{S-}$ & $\overline{S_{+}}$ & S- & $\overline{S-}$ & $\mathrm{S}_{+}$ & S- & $\overline{S-}$ & $S_{+}$ & S- & S- \\
\hline \multirow{10}{*}{ BOLO } & $\mathrm{BO}$ & TA & TU & 83 & 0 & 17 & 100 & & & 100 & & \\
\hline & & $\mathrm{TA}$ & VE & 100 & & & 89 & 0 & 11 & 67 & 33 & 0 \\
\hline & & $\mathrm{TA}$ & LA & 83 & 0 & 17 & 100 & & & 100 & & \\
\hline & & VE & LA & 67 & 17 & 17 & 100 & & & 100 & & \\
\hline & & $\mathrm{VE}$ & TU & 100 & & & 80 & 0 & 20 & 67 & 0 & 33 \\
\hline & $\mathrm{LO}$ & TA & TU & 67 & 0 & 33 & 67 & 33 & 0 & 67 & 0 & 33 \\
\hline & & $\mathrm{TA}$ & $\mathrm{VE}$ & 67 & 0 & 33 & 89 & 11 & 0 & 67 & 0 & 33 \\
\hline & & $\mathrm{TA}$ & LA & 67 & 33 & 0 & 100 & & & 100 & & \\
\hline & & $\mathrm{VE}$ & LA & 100 & & & 100 & & & 100 & & \\
\hline & & VE & TU & 67 & 17 & 17 & 86 & 0 & 14 & 100 & & \\
\hline \multirow{10}{*}{ TATU } & $\mathrm{TA}$ & $\mathrm{BO}$ & $\mathrm{LO}$ & 67 & 17 & 17 & 100 & & & 67 & 33 & 0 \\
\hline & & $\mathrm{BO}$ & $\mathrm{VE}$ & 50 & 0 & 50 & 67 & 22 & 11 & 67 & 33 & 0 \\
\hline & & $\mathrm{BO}$ & LA & 100 & & & 100 & & & 100 & & \\
\hline & & $\mathrm{VE}$ & LA & 67 & 17 & 17 & 56 & 33 & 11 & 33 & 0 & 67 \\
\hline & & VE & $\mathrm{LO}$ & 33 & 33 & 33 & 67 & 0 & 33 & 67 & 33 & 0 \\
\hline & $\mathrm{TU}$ & $\mathrm{BO}$ & LO & 33 & 33 & 33 & 67 & 0 & 33 & 67 & 33 & 0 \\
\hline & & $\mathrm{BO}$ & $\mathrm{VE}$ & 33 & 17 & 50 & 78 & 22 & 0 & 67 & 0 & 33 \\
\hline & & $\mathrm{BO}$ & LA & 0 & 83 & 17 & 78 & 11 & 11 & 33 & 33 & 33 \\
\hline & & VE & LA & 17 & 33 & 50 & 67 & 0 & 33 & 67 & 0 & 33 \\
\hline & & VE & LO & 83 & 17 & 0 & 100 & & & 33 & 33 & 33 \\
\hline \multirow{10}{*}{ VELA } & $\mathrm{VE}$ & $\mathrm{BO}$ & $\mathrm{LO}$ & 100 & & & 67 & 0 & 33 & 100 & & \\
\hline & & $\mathrm{BO}$ & $\mathrm{TA}$ & 67 & 17 & 17 & 67 & 33 & 0 & 67 & 33 & 0 \\
\hline & & $\mathrm{BO}$ & $\mathrm{TU}$ & 83 & 0 & 17 & 100 & & & 67 & 0 & 33 \\
\hline & & $\mathrm{TA}$ & $\mathrm{TU}$ & 67 & 17 & 17 & 67 & 33 & 0 & 67 & 33 & 0 \\
\hline & & $\mathrm{TA}$ & LO & 100 & & & 78 & 0 & 22 & 67 & 67 & 0 \\
\hline & LA & $\mathrm{BO}$ & LO & 67 & 33 & 0 & 100 & & & 100 & & \\
\hline & & $\mathrm{BO}$ & $\mathrm{TA}$ & 17 & 0 & 83 & 54 & 22 & 22 & 67 & 33 & 0 \\
\hline & & $\mathrm{BO}$ & TU & 67 & 33 & 0 & 100 & & & 100 & & \\
\hline & & $\mathrm{TA}$ & $\mathrm{TU}$ & 50 & 33 & 17 & 56 & 11 & 33 & 33 & 67 & 0 \\
\hline & & $\mathrm{TA}$ & LO & 17 & 67 & 17 & 67 & 0 & & 67 & 33 & 0 \\
\hline
\end{tabular}


Tabela 4

Porcentagem de escolha dos estímulos-comparação corretos e da escolha dos comparações incorretos com a primeira ou segunda sílaba da palavra apresentada como modelo nas tentativas SMTS palavra/ palavra do pré-treino e das tentativas SMTS palavra/palavra durante a fase de correção.

\begin{tabular}{|c|c|c|c|c|c|c|c|c|}
\hline \multicolumn{3}{|c|}{ Estímulos Comparação } & \multicolumn{3}{|c|}{$\begin{array}{l}\text { SMTS palavra/palavra } \\
\text { do pré-treino }\end{array}$} & \multicolumn{3}{|c|}{$\begin{array}{c}\text { SMTS palavra/palavra } \\
\text { durante o DOR }\end{array}$} \\
\hline$S_{+}$ & S- & S- & $S_{+}$ & $1^{\mathrm{a}}$ sílaba & $2^{\mathrm{a}}$ sílaba & $S_{+}$ & 1a sílaba & $2^{\mathrm{a}}$ sílaba \\
\hline BOLO & BOTU & TALO & 53 & 20 & 27 & 75 & 8 & 17 \\
\hline BOLO & BOLA & VELO & 53 & 20 & 27 & 45 & 55 & 0 \\
\hline BOLO & BOTU & VELO & 40 & 60 & 0 & 83 & 8 & 8 \\
\hline BOLO & BOLA & TALO & 33 & 60 & 7 & 75 & 20 & 10 \\
\hline $\mathrm{BOLO}^{*}$ & TALO & VELO & $93^{*}$ & $7^{*}$ & $0^{*}$ & 100 & & \\
\hline TATU & TALO & BOTU & 53 & 40 & 7 & 43 & 29 & 29 \\
\hline TATU & TALA & VETU & 67 & 27 & 7 & 43 & 43 & 14 \\
\hline TATU & TALO & VETU & 73 & 13 & 13 & 88 & 13 & 0 \\
\hline TATU & TALA & BOTU & 87 & 13 & 0 & 75 & 25 & 0 \\
\hline TATU* & BOTU & VETU & $80^{*}$ & $7^{*}$ & $13^{*}$ & 67 & 5 & 29 \\
\hline VELA & VELO & BOLA & 60 & 33 & 7 & 75 & 13 & 13 \\
\hline VELA & VETU & TALA & 80 & 13 & 7 & 80 & 7 & 13 \\
\hline VELA & VELO & TALA & 33 & 47 & 20 & 86 & 8 & 8 \\
\hline VELA & VETU & BOLA & 53 & 40 & 7 & 75 & 0 & 25 \\
\hline VELA* & BOLA & TALA & 73 & 13 & 13 & 78 & 6 & 17 \\
\hline
\end{tabular}

* Conjuntos de configuração que não respondem ao critério de ter a primeira sílaba do modelo em um estímulo-comparação incorreto e a segunda sílaba do modelo no outro comparação incorreto.

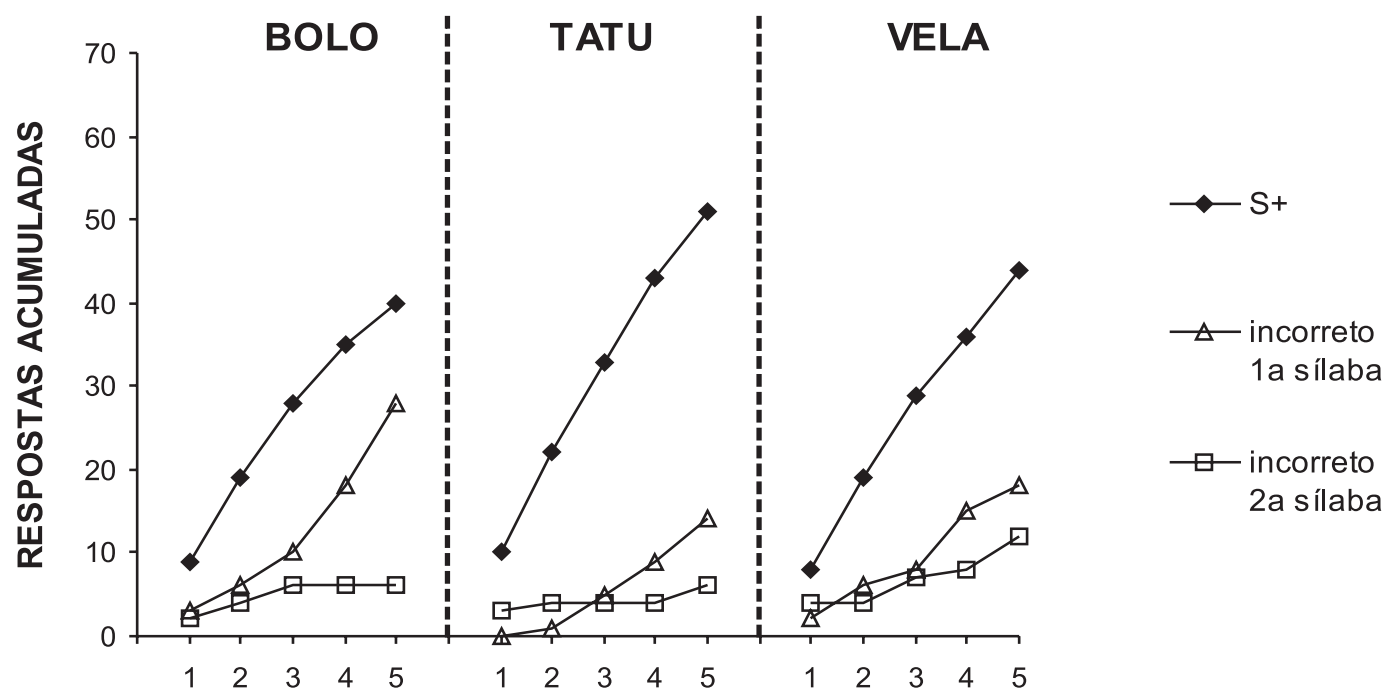

SESSÕES

Figura 3. Número acumulado de respostas de escolha do $S_{+}$, do comparação incorreto com a primeira sílaba da palavra apresentada como modelo e do comparação incorreto com a segunda sílaba da palavra apresentada como modelo nas cinco sessões do pré-teste de SMTS palavra/palavra. 
estímulo-comparação incorreto com a primeira sílaba do modelo e escolha do estímulocomparação incorreto com a segunda sílaba do estímulo-comparação incorreto. Cada gráfico da Figura 3 apresenta o número acumulado de respostas nos estímulos-comparação diante do estímulo-modelo especificado sobre as curvas. Podemos perceber um mesmo padrão de respostas ao longo das sessões: (a) maior número de acertos do que erros; (b) diminuição de respostas corretas; (c) aumento das respostas incorretas à medida que o participante é submetido às sessões. No geral, também houve um aumento gradativo de erros nos estímulos-comparação incorretos que contêm a primeira sílaba do estímulo-modelo.

$\mathrm{Na}$ Figura 4, aparece o mesmo tipo de análise da figura anterior para a tarefa SMTS palavra/palavra da condição DOR. A diferença principal é que na condição DOR o número de erros pela escolha do estímulocomparação incorreto com a segunda sílaba do modelo aproxima-se do número de erros pela escolha do estímulo-comparação incorreto com a segunda sílaba do modelo. No caso da palavra "VELA" houve mais erros pela escolha do estímulo-comparação incorreto com a segunda sílaba do modelo do que pela escolha do estímulo-comparação incorreto com a primeira sílaba. Podemos perceber um padrão de escolhas muito semelhante ao ocorrido na fase de pré-testes: a) número maior de acertos do que erros; b) diminuição de respostas corretas; c) aumento das respostas incorretas. Mais uma vez, o maior número de acertos ocorreu quando o estímulo-modelo era o "BOLO".

\section{DisCuSSĀo}

Controle restrito de estímulos é freqüentemente encontrado no desempenho de crianças com desenvolvimento atípico. $\mathrm{O}$ presente estudo teve como objetivo verificar se

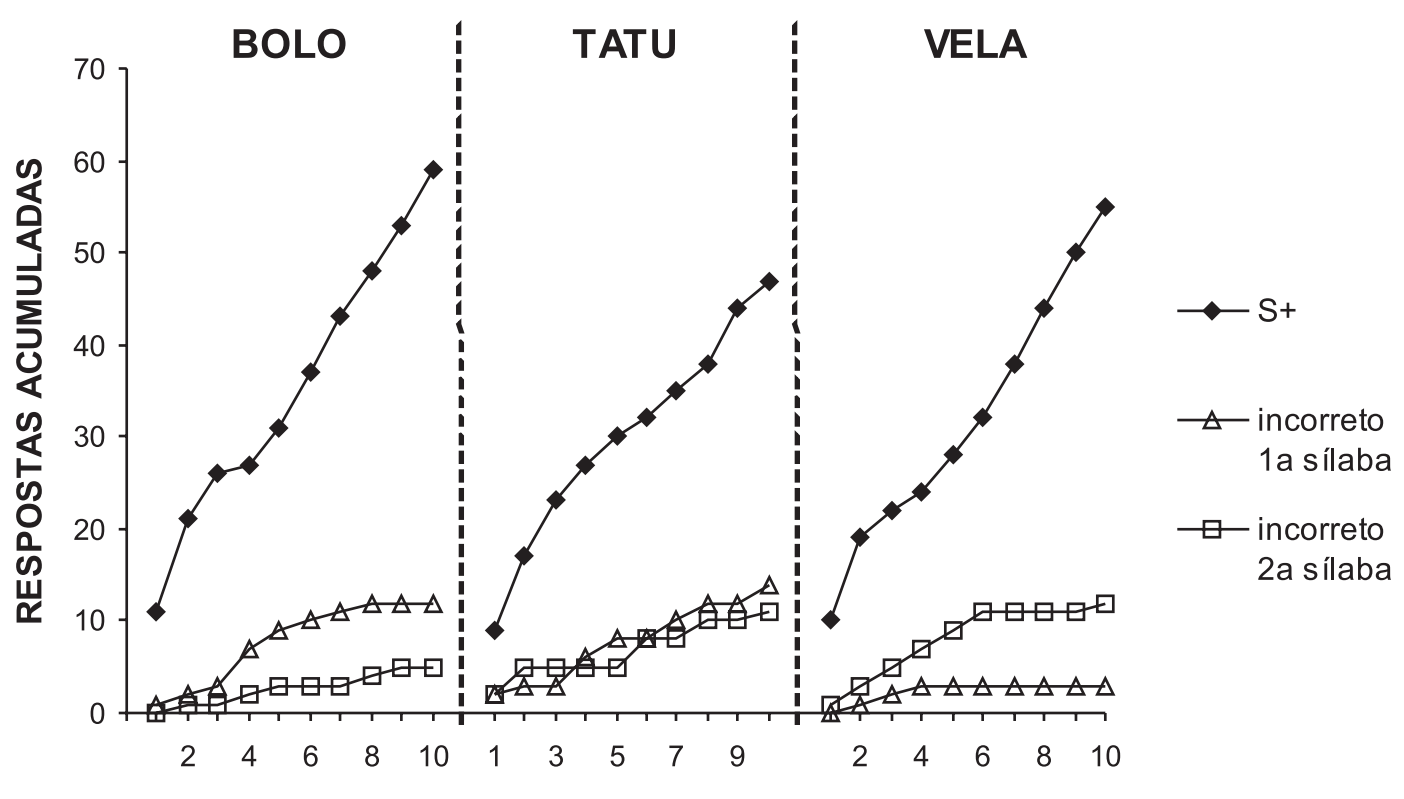

SESSÕES

Figura 4. Número acumulado de respostas de escolha do $S_{+}$, do comparação incorreto com a primeira sílaba da palavra apresentada como modelo e do comparação incorreto com a segunda sílaba da palavra apresentada como modelo nas tentativas SMTS palavra/palavra da condição de correção. 
era possível identificar e corrigir controle restrito de estímulos no desempenho de uma criança diagnosticada com autismo. As tarefas em que esse tipo de controle foi avaliado foram diferentes tarefas de MTS que utilizaram sílabas como estímulos simples, palavras como estímulos compostos e o procedimento chamado de resposta de observação diferencial para correção do controle restrito.

$\mathrm{Na}$ fase de pré-testes, os resultados indicaram que o participante não apresentou controle restrito de estímulos nas tarefas de SMTS e DMTS com sílabas. Já na tarefa de DMTS palavra/sílaba, o desempenho do participante indicou controle restrito de estímulos. Esses resultados assemelham-se aos do estudo de Dube e Mcllvane (1999) com estímulos compostos e simples.

A análise de erros das sessōes com palavras/ sílabas mostrou o mesmo padrão em tarefas de matching simultâneo palavra/sílaba: quando o estímulo modelo era o "TATU" e o comparação correto era o "TU", o participante escolhia a sílaba "LA" sempre que ela estava presente como comparação incorreto. Quando o estímulocomparação correto era a sílaba "TA", o participante escolheu o "LA" algumas vezes. É possível que o participante estivesse sob controle principalmente da letra "A" ao responder ao composto "TATU”. Essa hipótese se fortalece uma vez que esse padrão de erros se repetiu na tarefa de DMTS palavra/sílaba. Além disso, quando os estímulos-comparação eram atrasados em relação ao estímulo-modelo, ocorreu com muita freqüência a escolha do estímulo-comparação incorreto "TA" quando o estímulo-modelo era a palavra "VELA" e o estímulo-comparação correto a ser selecionado era a sílaba "LA" (isso também ocorreu com menor freqüência quando a sílaba correta a ser selecionada era o "VE"). Esse viés de erro nos permite dizer que ao responder ao estímulo "VELA", as escolhas posteriores do participante eram controladas principalmente pelo "VE" e depois da letra "A": quando o "VE" estava presente como estímulocomparação correto na configuração de estímulos, o participante escolhia-o na maioria das vezes e, portanto, acertava. Já quando havia os estímulos-comparação "LA" e "TA" simultaneamente, possivelmente apenas a letra "A" controlava sua resposta. Esses resultados mostram que pode ter havido controle restrito exercido por unidades menores que a sílaba, no caso, a letra. Evidencia adicional desse controle é o fato de que diante do modelo "BOLO" o participante apresentou, em todas as atividades, o menor número de erros. A diferença de "BOLO" em relação aos demais estímulos aconteceu porque o controle por "O" permitia acerto por responder a qualquer uma das sílabas.

Diferente do encontrado em Dube e Mcllvane (1999), os resultados do presente estudo mostraram a presença de controle restrito de estímulos na tarefa de matching simultâneo com palavras/palavras (estímulos compostos). Com a mudança do esquema CRF simples para o esquema de acúmulo de fichas, houve mais acertos. O controle de estímulos estabelecido pelas diferentes tarefas de matching pode ter interagido com uma variável motivacional: é possível que o item tangível que o participante escolhia no teste de preferências tenha, aos poucos, perdido o valor reforçador e, portanto, não tenha mantido o mesmo padrão de acertos de outras fases. Essa análise é coerente com a realizada por North e Iwata (2005), em que os autores demonstraram que acertos de participantes com 
desenvolvimento atípico diminuíam à medida que eram submetidos a reforçamento primário repetitivo, sugerindo a perda da efetividade reforçadora, uma vez que os itens preferidos eram consumidos com alta freqüência. No presente estudo, a perda do valor reforçador de itens escolhidos pelo participante dependeu possivelmente do grande número de tentativas em cada bloco durante as sessóes.

A análise de erros das sessões de SMTS palavra/palavra que indicaram controle restrito de estímulo permite a colocação da hipótese de que o participante estivesse sob controle principalmente da primeira sílaba do estímulo composto. O fato de ter havido desempenho acurado nas configurações nas quais justamente não havia estímulos-comparação incorretos com a primeira sílaba do modelo, dado o erro na programação no pré-teste, fortalece a hipótese de que o participante estava respondendo principalmente sob controle da primeira sílaba do estímulo composto apresentado como modelo, uma vez que a primeira sílaba do modelo estava presente somente no estímulo comparação correto

Em todas as etapas da Fase 2, linha de base, DOR e Pós-DOR, os níveis de precisão permaneceram em nível intermediário na maioria das sessões. Ocorreu aumento dos níveis de precisão nas sessões de Pós-DOR comparado à Linha de Base, mas não a ponto de podermos afirmar que o controle restrito foi eliminado. Mudanças no desempenho durante o procedimento DOR, contudo, puderam ser observadas. Durante o período de correção, houve duas sessóes em que o participante atingiu níveis muito altos de precisão, sugerindo a ausência de controle restrito de estímulos na tarefa de DMTS com palavra/sílaba. Apesar da instabilidade do padrão de respostas do participante após a introdução do DOR, nas tentativas de DMTS palavra/sílaba foram observados resultados que indicaram níveis acima daqueles obtidos na linha de base (com exceção da Sessão 5). Na maioria das sessóes de DOR (sete do total de dez), o nível de precisão ficou muito próximo ou superou o nível de precisão de $85 \%$. Esses resultados indicam que apesar de não ter eliminado completamente, a introdução do procedimento de DOR diminui o controle restrito.

Os resultados sugerem que o procedimento de DOR foi responsável por outras mudanças no controle de estímulos, mais sutis, que não puderam ser avaliadas pela medida mais geral de porcentagem de acerto. Um exemplo disso é o que foi demonstrado com o padrão de erros da fase de correção: a análise mostrou mudanças importantes no tipo de controle indesejado que pode caracterizar o controle restrito, como controle pela primeira sílaba do estímulo modelo versus controle pela segunda sílaba (o que pode ser visto na Tabela 4 e Figuras 3 e 4). Essa mudança de padrão mostra que o controle restrito é passível de ser modificado pelas contingências presentes nas tarefas de DOR.

Os resultados encontrados com o presente estudo corroboram parcialmente aqueles encontrados no estudo que serviu de base para a metodologia desta pesquisa (Dube e McIlvane, 1999). De fato, o desempenho do participante teve uma melhora após a introdução do procedimento de DOR. Além disso, o procedimento de DOR parece ter acarretado mudanças no que inicialmente foi identificado como controle restrito em uma tarefa com palavras.

Adicionalmente à discussão principal do presente estudo, os resultados sugerem a 
atualidade da proposta de Lovaas et al. (1979) sobre a relação entre controle restrito e o diagnóstico de autismo. Mostra, assim, a necessidade do desenvolvimento de estudos que identifiquem controle restrito de estímulos nos procedimentos que são comumente utilizados para o ensino de repertório comportamental. A identificação de como o controle restrito pode aparecer em certos procedimentos, como a tarefa de MTS com pessoas diagnosticadas com autismo, é um primeiro e importante passo na superação desse problema.

\section{REFERÊNCIAS}

Allen, K. D., \& Fuqua, R. W. (1985). Eliminating selective stimulus control: A comparison of two procedures for teaching mentally retarded children to respond to compound stimuli. Journal of Experimental Child Psychology, 39, 55-71.

Birnie-Selwyn, B., \& Guerin, B. (1997). Teaching children to spell: Decreasing consonant cluster errors by eliminating selective stimulus control. Journal of Applied Behavior Analysis, 30, 69-91.

DeLeon, I. G., \& Iwata, B. A. (1996). Evaluation of a multiple-stimulus presentation format for assessing reinforcer preferences. Journal of Applied Behavior Analysis, 29, 519-532.

de Rose, J. C., Souza, D. G., Rossito, A. L., \& de Rose, T. M. (1989). Aquisição de leitura após história de fracasso escolar: Equivalência de estímulos e generalização. Psicologia: Teoria e Pesquisa, 5, 325346.

Dube, W. V., Iennaco, F. M., \& McIlvane, W. J. (1993). Generalized identity matching to sample of twodimensional forms in individuals with intellectual disabilities. Research in Developmental Disabilities, 14, 457-477.

Dube, W. J., Lombard, K. M., Farren, K. M., Flusser, D. S., Balsamo, L. M., Fowler, T. R., \& Tomanari, G. Y.
(2003). Stimulus oversectivity and observing behavior in individuals with mental retardation. Em S. Soraci Jr. \& K. Murata-Soraci (Orgs.). Visual Information Processing (pp.109-124). Westport: Praeger Publishers.

Dube, W. V., \& McIlvane, W. J. (1999). Reduction of stimulus overselectivity with nonverbal differential observing responses. Journal of Applied Behavior Analysis, 32, 25-33.

Dube, W. V., \& Hiris, E. J. (1999). MTS software documentation. Waltham, MA: E. K. Shriver Center.

Hanna, E. S., de Souza, D. G., de Rose, J. C., \& Fonseca, M. (2004). Effects of delayed constructed-response indentity on spelling of dictated words. Journal of Applied Behavior Analysis, 37, 223-227.

Koegel, R. L., \& Koegel, L. K. (1995). Teaching Children with Autism. Baltimore: Paul H. Brookes Publishing Company.

Young, J. M., Krantz, P. J., McClannahan, L. E., \& Poulson, C. L. (1994). Generalized imitation and response-class formation in children with autism. Journal of Applied Behavior Analysis, 27, 685-697. LeBlanc, L. A., Coates, A. M., Daneshvar, S., CharlopChristy, M. H., Morris, C., \& Lancaster, B. M. (2003). Using video modeling and reinforcement to teach perspective-taking skills to children with autism. Journal of Applied Behavior Analysis, 36, 253-257.

Litrownik, A. J., McInnis, E. T., Wetzel-Pritchard, A. M., \& Filipelli, D. L. (1978). Restricted stimulus control and inferred attentional deficits in autistic and retarded children. Journal of Abnormal Psychology, 87, 554-562.

Lovaas, O. I., Schreibman. L., Koegel, R., \& Rehm, R. (1971). Selective responding by autistic children to multiple sensory input. Journal of Abnormal Psychology, 77, 211-222.

Lovaas, O. I., Koegel, R. L., \& Schreibman, L. (1979). Stimulus overselectivity in autism: a review of research. Psychological Bulletin, 86, 1236-1254. 
Matos, M. A., Hübner, M. M., \& Peres, W. (1997). Leitura generalizada: procedimento e resultados. Em R. A. Banaco (Org.), Sobre Comportamento e Cognição, Vol. 1 (pp 470-487), Santo André: Arbytes.

North, S. T., \& Iwata, B. A. (2005). Motivational influences on performance maintained by food reinforcement. Journal of Applied Behavior Analysis, 38, 317-333.

Peyton, R., Lindauer, S. E., \& Richman, D. M. (2005). The effects of directive and nondirective prompts on noncompliant vocal behavior exhibited by a child with autism. Journal of Applied Behavior Analysis, 38, 251-255.

Serna, R. W., McIlvane W. J., Lionello-DeNolf, K. M., Barros, R. S., \& Dube, W. V. (2000). Teoria de Coerência de Topografias de Controle de Estímulos na Aprendizagem Discriminativa: da pesquisa básica e Teoria à Aplicação. Em M. M. C. Hübner \& M. Marinotti (Orgs.), Análise do Comportamento para a Educação: Contribuiçôes Recentes (pp. 253-284). Campinas: ESETec

Thiemann, K. S., \& Goldstein, H. (2001). Social stories, written text cues, and video feedback: Effects on social communication of children with autism. Journal of Applied Behavior Analysis, 34, 425-446. Walpole, C. W., Roscoe, E. M., \& Dube, W. V. (2007). Use of a differential observing response to expand restricted stimulus control. Journal of Applied Behavior Analysis, 40, 707-712.

Submetido em 24 de julho de 2007 Aceito em 24 de fevereiro de 2008 


\section{Revista Brasileira de Análise do Comportamento \\ Brazilian Journal of Behavior Analysis}

\section{FORMULÁRIO DE ASSINATURA}

A Revista Brasileîra de Análise do Comportamento é uma publicaçāo semestral. Ao assiná-la você recebe os dois números publicados no ano. Números publicados anteriormente estóo também disponiveis.

Desejo receber (assinale):

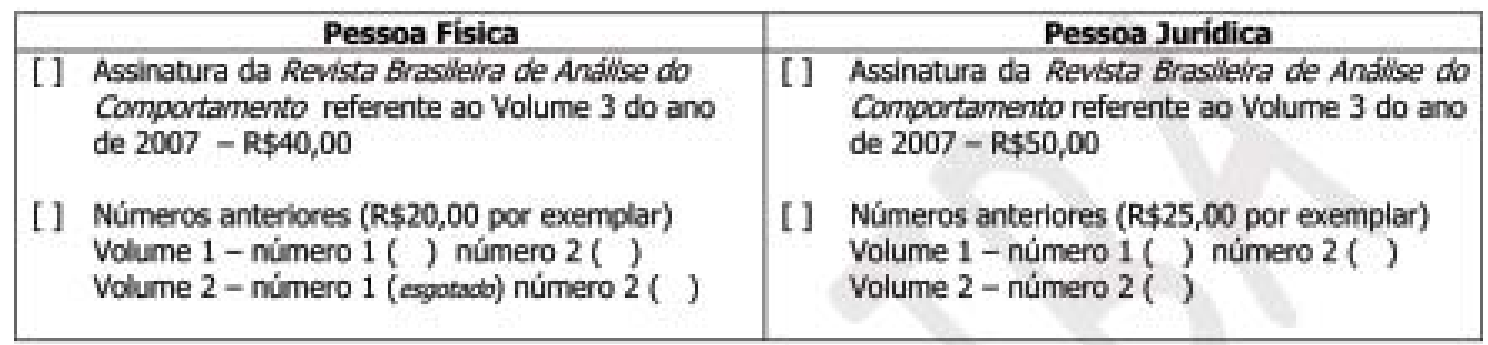

Entrega no exterior - taxa de correio: $R \$ 21,00$

\section{Forma de Pagamento}

[ ] Cheque nominal a CT-FUBRA ReBAC (Enviar cheque via correio, juntamente com esta ficha para o endereço ao lado).

[ ] Deposito no Banco do Brasil, ag. 3603-X, conta corrente 34.846-5 (Enviar o comprovante de depósito, juntamente com esta ficha por Fax, via correio ou por email)

Enviar para o endereco:

Revista Brasileira de Análise do Comportamento a.c.) Elenice Hanna (Editor)

Unimersidade de Brasilia

Instituto de Psicologia

Caixa Postal 4500

70.910-900 - Brasilia - DF

CNP]: 031.515.830.001-40

Ou via fax: $04+61 \quad 3273-0203$

Ou via email: assihantes rebaceigmall com

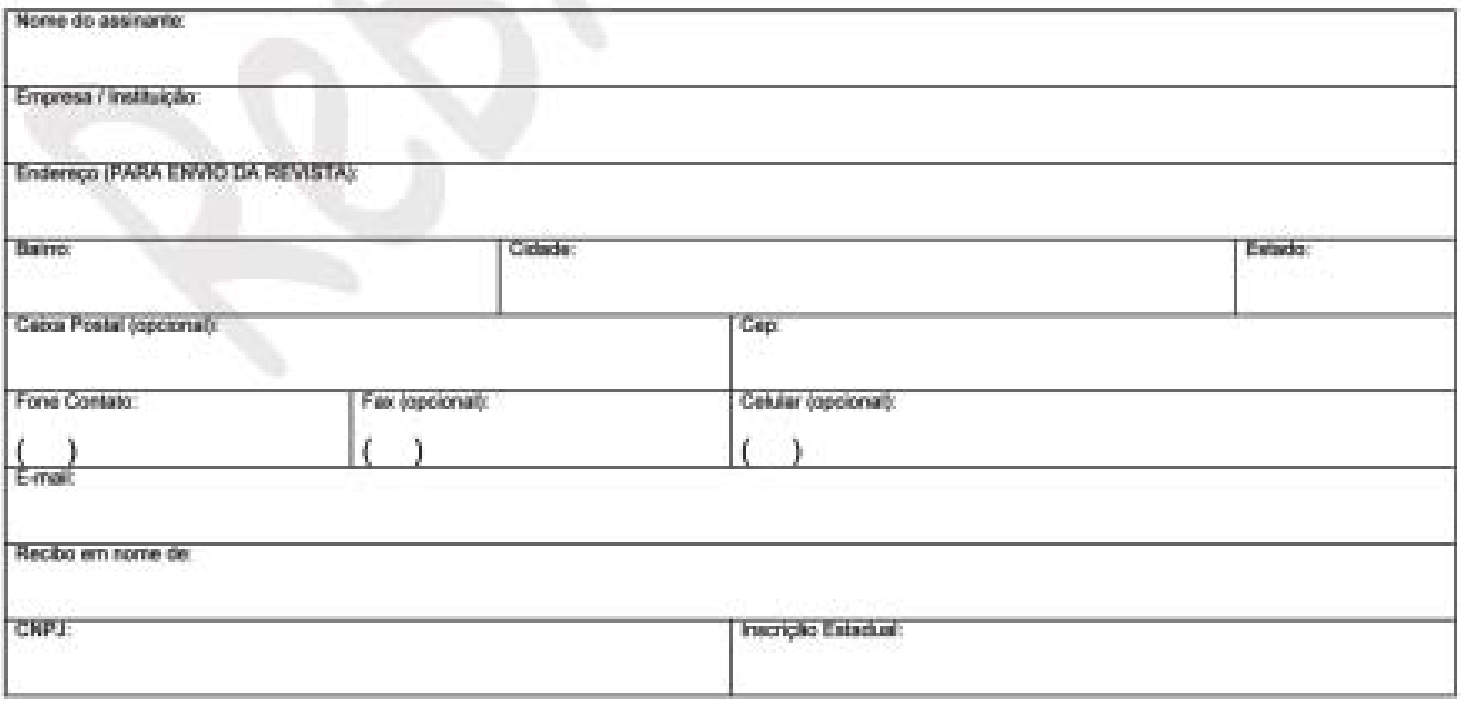

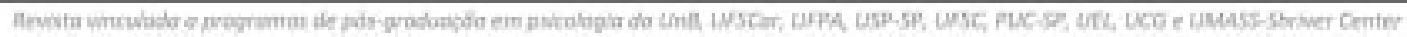

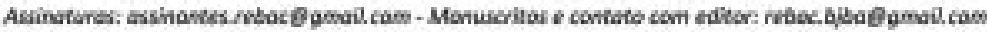

